\title{
Study on the Learning Adaptability and Mental Health of Wuhan College Students During the Outbreak of COVID-19
}

\author{
Hongxin Wang ${ }^{1}$, Jin $\mathrm{Xu}^{2}$, Liping Yang ${ }^{1}$, Saidi $\mathrm{Wan}^{3}$ \\ ${ }^{1}$ Evergrande School of Management, Wuhan University of Science and Technology, Wuhan, China \\ ${ }^{2}$ School of Computer Science and Technology, Wuhan University of Science and Technology, Wuhan, China \\ ${ }^{3}$ School of Foreign Language, Wuhan University of Science and Technology, Wuhan, China
}

Email address:

1084561041@qq.com (Jin Xu), 1936282091@qq.com (Hongxin Wang)

\section{To cite this article:}

Hongxin Wang, Jin Xu, Liping Yang, Saidi Wan. Study on the Learning Adaptability and Mental Health of Wuhan College Students During the Outbreak of COVID-19. Science Journal of Education. Vol. 8, No. 2, 2020, pp. 62-65. doi: 10.11648/j.sjedu.20200802.15

Received: April 18; Accepted: May 26, 2020; Published: June 3, 2020

\begin{abstract}
Objective: In this study to explore the relationships between learning adaptability and mental health of Wuhan college students during the epidemic period. Methods: This study was conducted among Wuhan college students in the form of questionnaire, with 484 valid data collected and processed by SPSS24.0 software. Descriptive statistics, independent-sample T-test, single factor analysis of variance, correlation analysis and regression analysis were adopted for this study. Results: (i) The learning adaptability of Wuhan college students was in the general level. (ii) These three variables, sex, region, grade, had no significant effect on learning adaptability. (iii) Learning adaptability was positively correlated with cognitive function, positive emotions and mental health while it was significantly negatively correlated with psychological symptoms and negative emotions. (iv) Eight percent change of learning adaptability (LA) could be interpreted by positive emotions (PE), cognitive function (CF) as well as psychological symptoms and negative emotions (PS\&NE). The formula for the relationship was: $\mathrm{LA}=1.55+0.06 * \mathrm{CF}+0.10 * \mathrm{PE}-0.05 * \mathrm{PS} \&$ NE. Conclusion: College students' learning adaptability which is not correlated with sex, region or grade, but highly related with mental health, is in the general level.
\end{abstract}

Keywords: Learning Adaptability, Mental Health, COVID-19, College Students

\section{Introduction}

At the end of 2019, a novel coronavirus (COVID-19), with high infectiousness and long incubation period, has quickly killed hundreds of people and sparked panic. As of $1 \mathrm{pm}$, April 6,2020, the authorities had inspected 1.27 million confirmed cases and 69549 deaths. To ensure the safety of people, many provinces in China have launched highest-level emergency response against the novel coronavirus and been sealed off from the outside world for two months. Now, as the situation in China is basically under control, many western countries have borrowed the policy of lockdown from China. Against this background, 850 million students around the world are forced to stay at home. The different teaching model, uncertain back-to-school date and long-time quarantine have not only led to students' poor mental health, but also dampened their enthusiasm to learn to a certain level [1].
Just like SARS, COVID-19 is highly contiguous. The policy of suspending classes without stopping teaching and learning followed the example of learning and teaching during holidays 17 years ago [2]. At that time, some experts had studied the relationship between learning adaptability and mental health of college students in the context of epidemic [3]. Xu Fuming and others investigated 723 college students in Tianjin by questionnaire to explore their pressure sources during the outbreak of SARS and the relationship between stress response strategies and mental health [4]. Through SPSS10.0 software, Chao Qiuling and others processed the data collected in the questionnaire, and explored the relationship between college students' learning adaptability and their mental health by using the high-low grouping method [5].

In this study, the main contributions are following: (i) We analyzed the whole level of college students' learning adaptability in the context of epidemic by descriptive statistics. (ii) We explored the effect of the three variables, sex, region 
and grade on the learning adaptability of college students by difference of examination. (iii) We explored whether learning adaptability correlate with the three dimensions of mental health as well as mental health by bi-variate correlation analysis. (iv) We explored the impact of three dimensions of mental health and mental health on learning adaptability by regression analysis.

\section{Object and Method}

\subsection{Research Object}

From March 26 to 31, 2020, we made a random survey of Wuhan college students who were voluntary and informed. We collected 484 valid questionnaires, among which, $43.60 \%$ were male, while $56.40 \%$ were female. $19.83 \%$ of the surveyed students were freshmen and $33.06 \%$ of them were sophomores while junior students and senior students accounted for $28.93 \%$ and $18.18 \%$ respectively. In regional level, $32.02 \%$ of them were from Hubei province while $67.98 \%$ were from other regions.

\subsection{Research Tool}

\subsubsection{Learning Adaptability Questionnaire for College Students}

The questionnaire referred to the "Learning Adaptability Scale for College Students" compiled by Feng Yanyong and others [6]. After collecting a lot of questionnaires, they analyzed the impact of learning motivation, learning attitude, learning ability, teaching mode and environmental factors on learning adaptability by exploratory factor analysis and confirmatory factor analysis. They induced and calculated thirteen factors whose characteristic values were greater than 1 , which could be used to explain $68.87 \%$ of the total variance. Learning motivation, learning attitude, learning ability, teaching mode and environmental factors had eight, four, six, seven and four items respectively, each item including five options which are "fully compliant", "more compliant", "uncertain", "less compliant" and "completely non-compliant". The classification of the four items composed of five classes $(0,1,2,3,4)$ stood for the level of satisfaction. The better learning adaptability indicated higher satisfaction level.

\subsubsection{Mental Health Questionnaire}

This questionnaire referred to the "Self-Rated Health Measurement Scale (SRHMS V1.0)" complied by Xu Jun [7], which measured a person's health in terms of psychology, physiology and society. The scale was composed of ten dimensions whose accumulative contribution rate accounted for $65.79 \%$. Our questionnaire included three items: cognitive function, positive emotions, psychological symptoms and negative emotions. Every item was scored as 0-5 points for the level of satisfaction. The higher scores of cognitive function and positive emotions indicated better mental health while the higher scores of psychological symptoms and negative emotions indicated worse mental health.

\subsection{Statistical Analysis}

All data were calculated by SPSS24.0 software.

\section{Results and Analysis}

\subsection{Learning Adaptability Level of College Students}

Firstly, in order to analyze the whole level of college students' learning adaptability, we adopted descriptive statistics method to process the 484 questionnaires. Table 1 shows the results. According to the table, most figures were fluctuating at the point of 1.95 that was slightly lower than 2 (theoretical median), indicating that college students' learning adaptability is in the general level in the context of COVID-19.

Table 1. Basic Statistical Value of Learning Adaptability.

\begin{tabular}{llll}
\hline & $\mathbf{n}$ & $\overline{\mathrm{X}} \pm s$ & TM \\
\hline $\mathrm{CF}$ & 484 & $1.95 \pm 0.47$ & 2 \\
\hline
\end{tabular}

*CF (cognitive function) TM (theoretical median)

Then, we adopted the difference of examination to explore whether sex, region and grade would have influence on college students' learning adaptability [8]. Table 2 shows the results.

Table 2. Difference of Examination.

\begin{tabular}{lllll}
\hline Item & & $\overline{\mathrm{X}}+\boldsymbol{S}$ & $\mathbf{P}$ \\
\hline \multirow{4}{*}{ Sex } & & & \\
& & male & $1.99 \pm 0.44$ & 0.05 \\
& female & $1.90 \pm 0.50$ & \\
& & & \\
RA & Hubei & $1.93 \pm 0.55$ & \multirow{2}{*}{0.59} \\
& & Non-Hubei & $1.96 \pm 0.42$ & \\
& \multirow{3}{*}{ Grade } & & & \\
& & Freshman & $1.91 \pm 0.43$ & \\
& & sophormore & $2.00 \pm 0.52$ & \multirow{2}{*}{0.16} \\
& & junior & $1.96 \pm 0.46$ & \\
& & senior & $1.88 \pm 0.42$ & \\
\hline
\end{tabular}

The results of independent sample t-test showed that no obvious difference was found in terms of sex and region for Wuhan college students [9]. According to the results by the method of one-way ANOVA, the learning adaptability of students in different grades varied slightly. These results indicated that the difference among Wuhan college students' learning adaptability was not obvious.

\subsection{Correlation Analysis of Learning Adaptability and Mental Health}

To explore the correlation of learning adaptability and mental health, we analyzed the correlation coefficient of learning adaptability and mental health as well as the correlation coefficient of three dimensions of mental health [10]. Table 3 shows the results. According to the table, learning adaptability of college students was positively correlated with cognitive function, positive emotions and mental health, but negatively correlated with psychological symptoms and negative emotions. 
Table 3. Correlation of Learning Adaptability and Mental Health.

\begin{tabular}{lllll}
\hline & CF & PS\&NE & PE & WH \\
\hline LA & $0.15^{* *}$ & $-0.18^{* *}$ & $0.23^{* *}$ & $0.27^{* *}$ \\
\hline
\end{tabular}

PE (positive emotions) MH (mental health)

*CF (cognitive function) LA (learning adaptability)

PS\&NE (psychological symptoms and negative emotions)

\subsection{Regression Analysis of Cognitive Function, Positive Emotions as Well as Negative Emotions \& \\ Psychological Symptoms and Learning Adaptability}

To give an insight into the impact of the three dimensions of mental health on learning adaptability, we used regression model to analyze the four variables, with cognitive function, positive emotions as well as psychological symptoms and negative emotions as the independent variables, and learning adaptability as the dependent variable. Table 4 shows the results. According to the table, the value of $\mathrm{R}^{2}$ was 0.08 , which indicated that the three dimensions of mental health accounted for eight percent change of learning adaptability. In this model, $\mathrm{F}=13.53, \mathrm{P}<0.001$, that meant at least one independent variable would affect learning adaptability. The significance analysis $(\mathrm{P}$ value $<0.05$ ) indicated that the three independent variables had a significant effect on learning adaptability. The $\mathrm{B}$ value of the three variables were $0.06,0.10,-0.05$ respectively, which indicated cognitive function and positive emotions had a positive correlation with learning adaptability while psychological symptoms and negative emotions had a negative correlation with learning adaptability. The formula for the relationship was: $\mathrm{LA}=$ $1.55+0.06 * \mathrm{CF}+0.10 * \mathrm{PE}-0.05 * \mathrm{PS} \& \mathrm{NE}$.

Table 4. Linear Regression Analysis.

\begin{tabular}{lllllll}
\hline & $\mathbf{U C}$ & & & $\mathbf{P}$ & $\mathbf{R}^{2}$ & $\mathbf{F}$ \\
\cline { 2 - 5 } & $\mathbf{B}$ & $\mathbf{S E}$ & & $\mathbf{T}$ & & \\
\hline Constant & 1.55 & 0.10 & 15.70 & $0.000^{* * *}$ & & \\
PE & 1.10 & 0.02 & 5.40 & $0.000^{* * *}$ & & \\
PS\&NE & -0.05 & 0.02 & -2.20 & $0.028 * * *$ & 0.08 & $13.53 * * *$ \\
CF & 0.06 & 0.02 & 3.08 & $0.002 * *$ & & \\
DV & LA & & & & & \\
$* * * \mathrm{P}<0.001, * * \mathrm{P}<0.05$ & & & & & \\
\hline
\end{tabular}

*UC (nonstandard coefficients) SE (standard Error)

PV (predictive variable) DV (dependent variable)

\subsection{Regression Analysis of Learning Adaptability and Mental Health}

To test whether mental health could predict learning adaptability, we made another regression analysis. Table 5 shows the results. On the basis of table, $\mathrm{F}=37.00, \mathrm{P}<0.001$, we can conclude that this model has forecasting significance. $\mathrm{B}=0.17, \mathrm{t}=6.08, \mathrm{P}<0.001$, that indicated that a person's mental health could positively predict his learning adaptability.

Table 5. Regression Analysis of Learning Adaptability and Mental Health.

\begin{tabular}{llllllll}
\hline IV & DV & B & SE & $\boldsymbol{\beta}$ & t & $\mathbf{R}^{2}$ & F \\
\hline LA & MH & 0.17 & 0.03 & 0.27 & $6.08 * * *$ & 0.07 & $37.00^{* * *}$ \\
\hline
\end{tabular}

*IV (independent variable) SE (standard Error) DV (dependent variable)

\section{Discussion}

The outbreak of this coronavirus did influence college students' studying and mental health to a certain level [11]. Online education is the only solution to suspending classes without stopping learning and teaching in light of long-period quarantine. Compared to offline education, online education is lack of interaction and good learning atmosphere. Most college students cannot make adjustment to the change of teaching model promptly and appropriately [12]. In addition, more free time together with the deficiency of supervision lead to students' procrastination habit and low learning efficiency [13]. Besides, network conditions, mobile terminal, learning tools and family atmosphere are uncertainties for college students [14]. Since the outbreak of the epidemic, many students have been affected by different negative emotions [15]. In our investigation, only less than 20 percent of college students had few negative emotions, which indicated this epidemic did influence college students' mental health. All the results above-mentioned suggest that developing a good habit of studying, stimulating learning motivation and keeping a good mood can improve one's learning adaptability.

\section{Conclusion}

Based on our study, the learning adaptability of Wuhan college students during the outbreak is in the general level regardless of their sexes, region or grades. In our study, college students' learning adaptability was significantly positively correlated with mental health. Our findings showed that college students' cognitive function, positive emotions and mental health had a positive correlation with learning adaptability while negative emotions and psychological symptoms had a negative effect on learning adaptability. In order to further explore the relationship between learning adaptability and the three dimensions of mental health, we made linear regression analysis. In the end, we regarded mental health as the predictive variable, learning adaptability as the dependent variable to test the reliability of the model. In accordance with our results, the regression model serves as a good predictor.

\section{References}

[1] Zhang Bin, Wang Ruili. The control strategy of college students psychological fluctuation in the outbreak of epidemic situation J. Educational exploration, 200912: 123-124.

[2] The General Office of the Ministry of Education: EB / Ol. HTP: www.moe. CN / S R s I t e / s 7056 / 202002 / t 202005418138. H TML, 2020 G 02G 02G 05

[3] Li Jun, a review of the research on learning adaptability of College Students J. China Science and education innovation guide, 2008, 31: 103, 105. Doi: 10.3969 J. ISSN. 1673-9795. 2008.31.087. 
[4] Xu Fuming, Yu Peng, Feng Hong. A study on the relationship between stress coping strategies and mental health of college students during the SARS epidemic period. Chinese Journal of Clinical Psychology, 200402: 163-164.

[5] Chao Qiuling, Xu Fuming, Huang Wenfeng, Wang Jingxin. Study on learning adaptability and its relationship with mental health of college students during the epidemic period of SARS J. Journal of Health Psychology, 200403: 209-211.

[6] Xu Jun, Albert Tam, Wang Yipeng, Hu minyan. Evaluation of SRHMS V1.0 revised self-rated health assessment scale. Chinese Journal of Mental Health, 200305: 301-305.

[7] Xu Fuming, Yu Peng, Feng Hong. A study on the mental health of college students in Tianjin during the SARS epidemic period J. School Health in China, 200404: 398-400.

[8] Shain Chak. A study on mental health and adaptability of college students J. Bulletin of science, education and culture, 201906: 156-157.

[9] Gao Jie, Du Yiming, Guo Xiuyue. An investigation of college students adaptability to online learning — An empirical analysis of online courses in Shaanxi Preschool Normal University. Journal of Shaanxi Preschool Normal University, 2019, 3509: 115-120.

[10] Song Fei-fei, Wang Xin, ju-young, Liu Aixiang, Liu Junjie, Wang Tao. A study on the mental health status of the public and its influencing factors during the epidemic period of 2019-ncov PNEUMONIA J. Public Health and Preventive Medicine, 2020, 3102: 23-27.

[11] Li Wenhao, Zhu zhiting. Improving emotional experience: J / $\mathrm{Ol}$ for relieving emotional problems of online learning during a pandemic. China's audio-visual education: 1-62020-05-08.

[12] $\mathrm{Hu}$ xiaoping, xie zuoyu. Analysis on the advantages and challenges of online teaching in universities under the epidemic situation [J]. China higher education research, 2020 (04): 18-22 58.

[13] Zhang Yaning, Peng Haihao. Mediation effect of time management disposition on self-consciousness and learning adaptability of college students — A case study of mental health of freshmen in a college of traditional Chinese medicine. Journal of Social Sciences of Shanxi University, 2020, 3201: 47-51.

[14] Peng Yanzhen. A study on the relationship between family cohesion, adaptability and mental health of junior middle school students. Hunan University of Science and Technology, 2017.

[15] Zhao Bo, Clara Zetkin, Zhang Zhihua. The presentation and adjustment of college students'emotional state in the epidemic of new pneumonia -- from the perspective of self-care. China Youth Studies, 20204: 49-54. 